\title{
BMSAP
}

Bulletins et mémoires de la Société d'Anthropologie de Paris

BMSAP

$33(1) \mid 2021$

Les 1000 premiers jours de vie dans les populations du présent et du passé

\section{Malnutrition infantile à la fin de la période laténienne : l'apport de l'examen paléopathologique de la nécropole de Jort (Calvados, Normandie, France)}

Infant malnutrition in the late La Tène period: Contribution of the

palaeopathological examination of the Jort necropolis (Calvados, Normandy,

France)

Frédéric Boursier, Raphaëlle Lefebvre, Denis Bougault, Cécile Chapelain de Séreville-Niel, Dominique Corde et Vincent Carpentier

\section{(2) OpenEdition}

Édition électronique

URL : https://journals.openedition.org/bmsap/7445

DOI : $10.4000 /$ bmsap. 7445

ISSN : $1777-5469$

Éditeur

Société d'Anthropologie de Paris

Référence électronique

Frédéric Boursier, Raphaëlle Lefebvre, Denis Bougault, Cécile Chapelain de Séreville-Niel, Dominique

Corde et Vincent Carpentier, « Malnutrition infantile à la fin de la période laténienne : l'apport de l'examen paléopathologique de la nécropole de Jort (Calvados, Normandie, France) », Bulletins et mémoires de la Société d'Anthropologie de Paris [En ligne], 33 (1) | 2021, mis en ligne le 27 avril 2021, consulté le 18 mars 2022. URL : http://journals.openedition.org/bmsap/7445 ; DOI : https://doi.org/ 10.4000/bmsap.7445

Les contenus des Bulletins et mémoires de la Société d'Anthropologie de Paris sont mis à disposition selon les termes de la licence Creative Commons Attribution-NonCommercial-NoDerivatives 4.0 International License. 


\title{
Malnutrition infantile à la fin de la période laténienne : l'apport de l'examen paléopathologique de la nécropole de Jort (Calvados, Normandie, France)
}

\author{
Infant malnutrition in the late La Tène period: Contribution of the palaeopathological \\ examination of the Jort necropolis (Calvados, Normandy, France)
}

Frédéric Boursier (iD 1,2*, Raphaëlle Lefebvre ${ }^{3}$, Denis Bougault (iD) ${ }^{3}$,

Cécile Chapelain de Séreville-Niel (D) ${ }^{3}$, Dominique Corde ${ }^{3}$, Vincent Carpentier ${ }^{3,4}$

1 Centre Hospitalier de Gonesse, Val-d'Oise, France

2 UMR 7206 Eco-Anthropologie (EA), MNHN-CNRS-Université de Paris Diderot, Musée de l'Homme, France

3 UMR 6273 CRAHAM, Centre Michel de Boüard, Université de Caen-CNRS, Caen, France

4 INRAP Grand Ouest

* frederic.boursier@ch-gonesse.fr

Reçu : 15 avril 2020 ; accepté : 17 novembre 2020 Bulletins et Mémoires de la Société d'Anthropologie de Paris

Cette note fait suite à une communication présentée lors des $1845^{\text {es }}$ journées de la Société d'Anthropologie de Paris dans le cadre de la session "Les 1000 premiers jours de vie dans les populations du présent et du passé"

Résumé - Les deux campagnes de fouilles de la nécropole de Jort (Calvados), datant de La Tène finale, ont permis de mettre en évidence un site exceptionnel par son recrutement funéraire, constitué majoritairement d'enfants de moins de cinq ans. Cette concentration d'immatures est une découverte inédite pour cette période et dans la région Normandie, ne trouvant aucune explication évidente à ce jour. L'examen paléopathologique de notre collection met en évidence une forte récurrence de lésions pathologiques décrites comme associées à des phénomènes carentiels. La population de Jort présente ainsi un profil paléopathologique pouvant s'inscrire dans une situation de famine qui est à mettre en rapport avec la grande instabilité sociale de la fin de La Tène.

Mots clés - second Âge du Fer, scorbut, malnutrition

Abstract - The two archaeological campaigns to excavate the Jort necropolis (Calvados, France), dating to the La Tène D period, revealed a site of exceptional interest for the large number of immature individuals, of which the majority was under five years old. This concentration of immature individuals is unique for this period and region, and as yet there is no obvious explanation accounting for it. The palaeopathological examination of our collection showed a significant recurrence of pathological lesions that are usually associated with deficiency diseases. This population at Jort thus displays a palaeopathological profile potentially indicating a famine situation that could have been related to the serious social instability that characterised the end of the La Tène culture.
Keywords - second Iron Age, scurvy, malnutrition

\section{Introduction}

La transition La Tène / période gallo-romaine débute par le conflit césarien et aboutit à une véritable acculturation 50 ans plus tard, pouvant être lue comme une crise sociale majeure pour la société celtique du Nord de la Gaule. Longtemps considérée comme une phase de transition acceptée et acceptable pour les populations, l'archéologie met de plus en plus en évidence les traces d'une présence militaire, concordant avec les épisodes de révoltes rapportés par les auteurs latins, reflétant la violence sociale à l'origine de la romanisation (Feugère et Poux, 2001 ; Le Roux, 2004 ; Reddé, 2018). Les conséquences anthropologiques de cette transition n'ont pas fait l'objet d'études spécifiques, laissant ainsi un doute sur la réalité et l'ampleur de la crise. Les effets supposés d'une colonisation violente seraient une augmentation des violences interpersonnelles, des troubles des circuits de l'alimentation, à l'origine de famines, et favorisant les épisodes épidémiques. La rareté des sites datés de cette période peut avoir favorisé l'absence de mise en évidence d'éléments évocateurs de troubles. La nécropole de Jort, datée de La Tène D1/D2 au début de la période gallo-romaine, présente un recrutement en jeunes immatures inhabituel, permettant d'aborder l'état sanitaire d'un échantillon populationnel infantile. L'importance des moins de 5 ans (93\%) parmi les 105 sujets et la récurrence, chez ces derniers, d'un ensemble de lésions fréquemment retrouvées en contexte carentiel avéré, nous amène à discuter d'une situation de malnutrition infantile possible. 


\section{Contexte archéologique}

\section{Présentation générale}

Le site de Jort "Rue Paul-Duhomme" est localisé au sud-est de Caen. Deux fouilles effectuées sur des parcelles séparées ont permis de mettre au jour des vestiges échelonnés du Néolithique ancien jusqu'à l'Époque moderne (Carpentier, 2015). L'occupation la plus conséquente est une nécropole datée de La Tène finale (D1 et D2) comptabilisant à ce jour environ 135 sépultures dont 29 vides.

\section{Pratiques funéraires}

Excepté deux incinérations, toutes les tombes sont des inhumations individuelles primaires, relativement dispersées dans l'espace, et sans aucun élément fédérateur (monument, tombe remarquable). Aucun recoupement entre les fosses n'a été observé, ce qui pourrait témoigner de leur visibilité dans l'espace tout au long de la durée d'occupation de la nécropole. Aucune sectorisation en fonction de l'âge n'est observée. Les fosses sépulcrales suivent deux orientations principales : un axe majoritairement nord-sud, avec de légères variations, ou un axe strictement ouest-est. La place de la tête du défunt n'est pas normalisée puisqu'elle se trouve à l'une ou l'autre des extrémités. La position des corps est assez homogène. 56 défunts ont été déposés sur le dos, 5 sont inhumés sur le côté, et 3 en procubitus. Les membres supérieurs sont placés préférentiellement le long du corps, et les membres inférieurs sont en extension, avec parfois les jambes croisées, ou en flexion. Cette hétérogénéité des positions de dépôt, est déjà rencontrée sur plusieurs autres sites normands au cours de la Tène finale, comme à Urville-Nacqueville (Lefort et Rottier, 2014) ou Ifs (Besnard-Vautrin, 2011).

La conservation relativement médiocre des ossements a limité l'étude taphonomique et la détermination des modes d'inhumation. Seule la présence de mobilier directement lié à l'architecture funéraire ou au port de vêtements, en a permis l'identification. 14 contenants en bois ont été reconnus (présence de clous ou de crampons en fer). Plusieurs fibules et un élément de ceinture en position fonctionnelle attestent de l'existence probable de vêtements ou d'une enveloppe souple dans 21 inhumations. Actuellement, 30 tombes ont un dépôt de mobilier funéraire avec des éléments de parure (fibules, bracelets, perles). Des offrandes ont été découvertes par le biais de dépôts céramiques généralement déposés à la tête du défunt. Enfin, une petite clochette en alliage cuivreux, objet souvent déposé dans les tombes à des fins apotropaïques, a également été retrouvée. Ces jeunes défunts de Jort sont dotés de parures et de mobiliers comparables à ceux des adultes découverts dans d'autres cimetières normands de la même époque (Chanson et al., 2010).

\section{Méthodes}

L'examen initial réalisé à la suite des fouilles avait indiqué une fréquence élevée d'immatures présentant des lésions osseuses d'aspect pathologique. Face à une surreprésentation d'immatures et un ensemble de signes évoquant une forte morbidité, la question était de savoir si ces constatations pouvaient être rattachées à un unique cadre nosologique, et ainsi entrevoir un ensemble étiologique cohérent, particulièrement celui de la malnutrition infantile.

Pour aborder l'état sanitaire de cette population, nous avons donc choisi de réaliser un profil paléopathologique basé sur les lésions observées plutôt qu'un profil par diagnostics de forte probabilité, qui aurait rejeté de fait les diagnostics différentiels. Un cumul de diagnostics individuels, dans un contexte de paléopathologie d'immatures, ne nous a pas semblé pertinent compte tenu de la faible sensibilité des lésions pathologiques chez l'enfant.

La malnutrition n'est pas un diagnostic paléopathologique individuel possible. S'il est démontré que les carences alimentaires ont une conséquence forte sur la croissance et le développement de la corticale osseuse, ces symptômes ne sont pas évaluables en contexte d'anthropologie funéraire (Garn et al., 1969 ; Vis, 1969). L'histoire de l'humanité est marquée par des famines, et les épisodes récents montrent que les jeunes enfants sont les plus touchés, avec des causes de décès bien identifiées, principalement d'origine infectieuses, qui encore une fois ne sont pas identifiables par les paléopathologistes (Toole et Waldman, 1988). La littérature paléopathologique retient des situations de malnutrition quand s'associe, à un contexte historique probant, la présence de lésions osseuses carentielles, notamment le scorbut ainsi que le rachitisme (Geber et Murphy, 2012).

Le rachitisme, par les déformations osseuses qu'il entraine, présente peu de difficulté de diagnostic. Plus que la conséquence d'une carence alimentaire, le manque de vitamine $\mathrm{D}$, principalement synthétisé par la peau, est un marqueur de paupérisation, particulièrement important à l'Époque moderne (Brickley et al., 2017). Le scorbut présente des difficultés diagnostics importantes. Ortner décrit comme lésions principales les atteintes, à type de porosités ou d'appositions périostées, des grandes ailes du sphénoïde, ainsi que celles des toits des orbites, mais d'autres localisations sont proposées au niveau de l'exocrâne, du maxillaire, de la mandibule, du palais, de l'os zygomatique, de la scapula, et des extrémités métaphysaires des os longs (Ortner et al., 2001). Pour Ortner, ce n'est pas tant l'aspect, décrit comme "porous lesions", ou comme "hypertrophic bone formation", qui oriente le diagnostic, que la localisation des lésions, toujours en association à un contexte historique évocateur. Ces signes ont fait l'objet de réévaluations, montrant ainsi qu'ils peuvent être présents dans d'autres pathologies (traumatisme, infection, néoplasie) voire dans des conditions physiologiques, soulignant leurs caractères non-pathognomoniques, en dehors des grandes ailes du sphénoïde (Lewis, 2017 ; Buikstra, 2019). La revue de la littérature des signes rapportés au scorbut, réalisée par Snoddy, propose un classement en diagnostics, évocateurs ou absence de critères évocateurs, en fonction du niveau de preuve, sans pouvoir proposer une grille de diagnostic, laissant ainsi la discussion diagnostique à chacun (tableau 1 ; Snoddy et al., 2018). 


\begin{tabular}{|c|c|c|c|}
\hline Localisation & Description des lésions (Snoddy 2018) & Classification & $\begin{array}{l}\text { Nombre } \\
\text { d'immatures }\end{array}$ \\
\hline \multirow{3}{*}{ Calvarium } & $\begin{array}{l}\text { Porous subperiosteal new bone on the ectocranial surface } \\
\text { of the parietal and/or squamous temporal bones }\end{array}$ & Diagnostic & 7 \\
\hline & $\begin{array}{l}\text { Subperiosteal new bone with vascular impressions and/or fine cortical porosity } \\
\text { on the endocranial surface of the vault }\end{array}$ & Suggestive & 4 \\
\hline & $\begin{array}{l}\text { Abnormal cortical porosity and/or subperiosteal new bone } \\
\text { in the orbital roofs/lateral orbit }\end{array}$ & Diagnostic & 7 \\
\hline \multirow{3}{*}{ Maxillae } & $\begin{array}{l}\text { Abnormal cortical porosity and/or porous subperiosteal new bone } \\
\text { on the anterior surface and/or in the region of the infraorbital foramina }\end{array}$ & Diagnostic & 0 \\
\hline & $\begin{array}{l}\text { Abnormal cortical porosity and/or porous subperiosteal new bone } \\
\text { on the posterior surface, superior to the alveolar margins }\end{array}$ & Diagnostic & 3 \\
\hline & $\begin{array}{l}\text { Abnormal cortical porosity and/or porous subperiosteal new bone } \\
\text { on the maxillary palate, outside the alveolar margins }\end{array}$ & Diagnostic & 1 \\
\hline \multirow{2}{*}{ Zygomata } & $\begin{array}{l}\text { Abnormal cortical porosity and/or subperiosteal new bone } \\
\text { on the lateral portion of the anterior surface }\end{array}$ & Suggestive & 1 \\
\hline & Abnormal cortical porosity on the internal/posterior surface & Suggestive & 0 \\
\hline \multirow{4}{*}{ Sphenoid } & Abnormal cortical porosity on the external surface of the greater wing & Diagnostic & 3 \\
\hline & Subperiosteal new bone around the foramen rotundum & $\begin{array}{l}\text { Tentatively } \\
\text { diagnostic }\end{array}$ & 1 \\
\hline & Abnormal cortical porosity on the lesser wings & Suggestive & 2 \\
\hline & $\begin{array}{l}\text { Abnormal cortical porosity and/or subperiosteal new bone } \\
\text { in the pterygoid fossae }\end{array}$ & Diagnostic & 0 \\
\hline Occipital & $\begin{array}{l}\text { Abnormal cortical porosity on the inferior surface of pars basilaris (juveniles } \\
\text { only) }\end{array}$ & Suggestive & 6 \\
\hline \multirow{2}{*}{ Mandible } & $\begin{array}{l}\text { Abnormal cortical porosity and/or subperiosteal new bone } \\
\text { on the interior surface of the rami/coronoid process }\end{array}$ & Diagnostic & 6 \\
\hline & $\begin{array}{l}\text { Subperiosteal new bone along the mylohyoid line } \\
\text { on the interior surface of the body }\end{array}$ & Diagnostic & 0 \\
\hline Scapula & $\begin{array}{l}\text { Abnormal cortical porosity and/or subperiosteal new bone } \\
\text { in the supra and/or infraspinous fossae }\end{array}$ & Diagnostic & 1 \\
\hline \multirow{2}{*}{ Ribs } & Enlargement of the costochrondral junction (juveniles only) & Suggestive & 0 \\
\hline & Subperiosteal new bone on the anterior/lateral surfaces of the shafts & Suggestive & 1 \\
\hline Vertebrae & Biconcavity and osteopenia of bodies (adults only) & Suggestive & 0 \\
\hline Ilia & $\begin{array}{l}\text { Abnormal cortical porosity and/or subperiosteal new bone, particularly } \\
\text { on the visceral surface. Subperiosteal new bone may have vascular impressions }\end{array}$ & Suggestive & 7 \\
\hline \multirow{3}{*}{$\begin{array}{l}\text { Appendicular } \\
\text { skeleton }\end{array}$} & $\begin{array}{l}\text { Widespread, porous, subperiosteal new bone on the diaphyses/metaphyses } \\
\text { of long bones (adults and juveniles) }\end{array}$ & $\begin{array}{l}\text { Suggestive if } \\
\text { bilateral }\end{array}$ & 13 \\
\hline & Discrete ossified haematomas & Suggestive & 0 \\
\hline & Metaphyseal cupping/flaring (juveniles) & Suggestive & 0 \\
\hline Femora & $\begin{array}{l}\text { Subperiosteal new bone on the proximal and medial diaphysis, medial to the } \\
\text { linea aspera }\end{array}$ & Suggestive & 10 \\
\hline
\end{tabular}

Tableau 1. Répartition des atteintes évocatrices du scorbut chez les immatures pathologiques de Jort (n=36) d'après Snoddy, 2018 / Distribution of scurvy criteria in diseased children in the Jort population ( $n=36)$, Snoddy 2018 
Dans ce cadre, l'établissement du diagnostic de scorbut peut devenir complexe, surtout si le contexte historique n'est pas assuré. En conséquence, nous avons décidé de réaliser un profil paléopathologique par lésion, qui permet de discuter les différentes hypothèses diagnostiques et étiologiques. C'est l'écart à la norme qui peut permettre d'entrevoir une répartition naturelle des pathologies attendues dans une population d'enfants décédés en bas âge, et d'apporter un élément de preuve supplémentaire pour retenir une étiologie. En pratique, l'examen a été réalisé en plusieurs étapes successives :

- l'évaluation de l'état de conservation des squelettes, de manière à mesurer la lisibilité des lésions pathologiques. Pour chaque individu examiné, la qualité de conservation du squelette et celle du tissu ostéo-archéologique et de la surface corticale ont été mesurées, puis classées en 4 catégories : bonne (entre 100 et $75 \%$ ), moyenne (entre 75 et $50 \%$ ), mauvaise (entre 50 et $25 \%$ ) et très mauvaise (entre 25 et $1 \%$ ) (Bello et al., 2002);

- l'estimation de l'âge au décès des immatures d'après la méthode métrique d'Adalian (2001) ou la méthode dentaire de Moorrees et al. (1963). Sept groupes d'âge ont été retenus (figure 1) et une ventilation des individus à cheval sur deux classes d'âge a été réalisée ;

- enfin, un examen macroscopique systématique de toutes les pièces osseuses a été réalisé, en colligeant, pour chaque individu, l'ensemble des lésions osseuses pathologiques et leurs localisations.

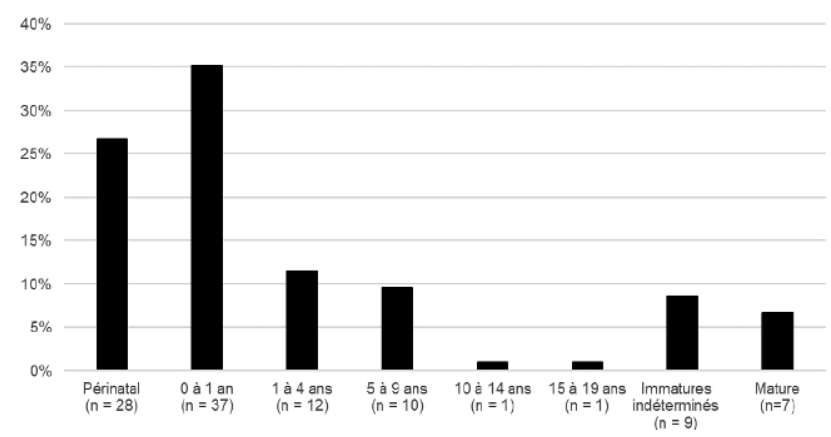

Figure 1. Répartition par âge de la population de Jort / Age distribution of the Jort population

\section{Résultats}

La collection ostéologique issue des fouilles de Jort est marquée par une forte représentation des plus jeunes. Parmi les 105 individus exhumés, 98 sont de très jeunes immatures avec $73 \%$ de moins de 5 ans et $27 \%$ de périnatals (figure 1).

Cette importance des immatures peut expliquer la faible représentativité ostéologique et la mauvaise conservation osseuse. Trois quarts des individus avaient un taux de représentativité osseuse inférieur à $75 \%$ et $80 \%$ des squelettes avaient une altération majeure de la corticale (supérieure à $50 \%$ ). L'examen paléopathologique s'en est trouvé limité car de nombreuses pièces osseuses sont absentes ou trop altérées pour fournir des informations.

Malgré cet écueil, l'examen paléopathologique a mis en évidence que $39 \%$ de la population présente au moins une lésion osseuse pathologique, et $36 \%$ pour les immatures. Ces lésions apparaissent sous la forme de porosité osseuse anormale ( $12 \%$ des immatures), et/ou d'appositions périostées (33\% des immatures). $17 \%$ des enfants atteints ne présentent qu'un seul site lésé, $39 \%$ présentent deux sites et $44 \%$ plus de trois sites, allant jusqu'à dix sites pour le cas le plus extrême. Nous constatons que toutes les classes d'âge sont concernées (figure 2). La répartition anatomique des lésions pathologiques montre une prédominance sur les os longs des membres supérieurs et inférieurs ainsi qu'au niveau du massif facial (tableau 2). Proposer un diagnostic individuel sur ce type de lésions, peu spécifiques, est ardu.

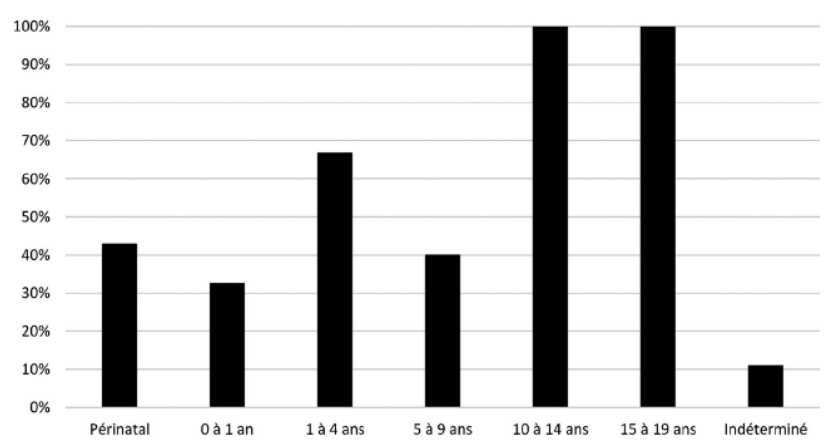

Figure 2. Proportion de lésions pathologiques (porosités/ appositions périostées) par classe d'âge d'immatures / Proportion of pathological lesions (porous lesions/new bone formation) by child age groups

\begin{tabular}{|c|c|}
\hline Localisation des lésions & $\begin{array}{c}\text { Proportion } \\
\text { d'immatures atteints }\end{array}$ \\
\hline Os Long & $\mathbf{1 9 \%}$ \\
\hline Fémur & $11 \%$ \\
\hline Humérus & $7 \%$ \\
\hline Tibia & $7 \%$ \\
\hline Os de l'avant-bras & $2 \%$ \\
\hline Région faciale & $\mathbf{1 6} \%$ \\
\hline Plafond de l'orbite & $8 \%$ \\
\hline Mandibule & $5 \%$ \\
\hline Maxillaire & $3 \%$ \\
\hline Os zygomatique & $1 \%$ \\
\hline Voute du crâne & $\mathbf{1 0} \%$ \\
\hline Exocrâne & $6 \%$ \\
\hline Endocrâne & $4 \%$ \\
\hline Base du crâne & $\mathbf{1 0} \%$ \\
\hline Os sphénoïde & $5 \%$ \\
\hline Pars basilaris & $5 \%$ \\
\hline Autres localisations & $\mathbf{1 0} \%$ \\
\hline Os Coxal & $7 \%$ \\
\hline Côtes & $1 \%$ \\
\hline Scapula & $1 \%$ \\
\hline
\end{tabular}

Tableau 2. Répartition anatomique des lésions pathologiques (porosités/appositions périostées) / Anatomical distribution of pathological lesions (porous lesions/new bone formation) 


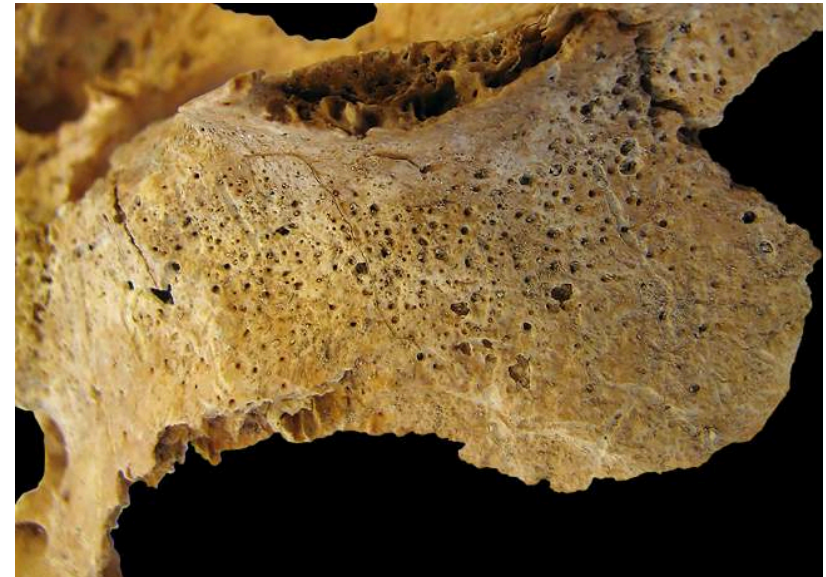

Figure 3. Grande aile du sphénoïde gauche, individu 38 (âgé entre 5 et 9 ans) / Left greater wing of the sphenoid bone, skeleton 38 (5-9 year-old child)

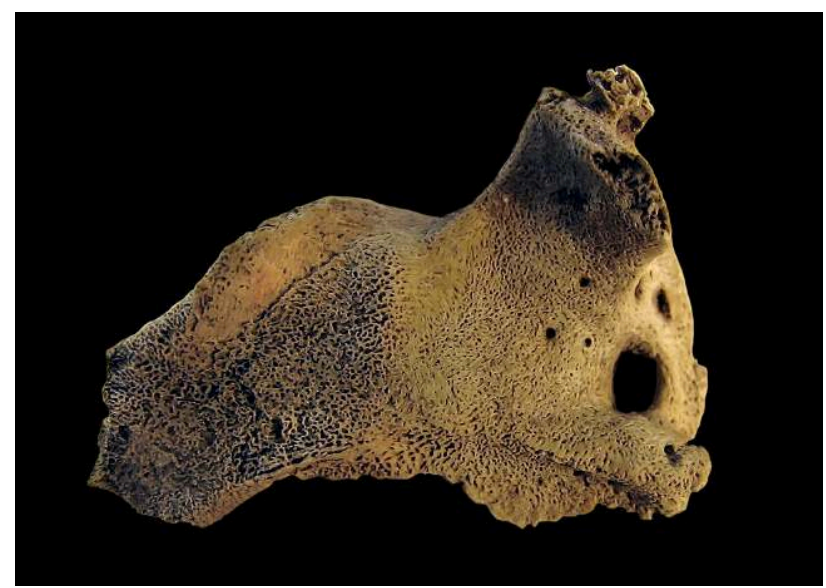

Figure 4. Grande aile du sphénoïde droit, individu 132, périnatal / Right greater wing of sphenoid bone, skeleton 132, perinatal

\begin{tabular}{|c|c|}
\hline Types de critères & $\begin{array}{c}\text { Proportion d'immatures } \\
\text { pathologiques }(\mathbf{n}=\mathbf{3 6})\end{array}$ \\
\hline $\begin{array}{c}\text { Plusieurs critères diagnostics } \\
\text { +/- des critères évocateurs }\end{array}$ & $28 \%$ \\
\hline $\begin{array}{c}\text { Un critère diagnostic }+ \\
\text { un ou plusieurs évocateurs }\end{array}$ & $14 \%$ \\
\hline Un critère diagnostic & $11 \%$ \\
\hline Plusieurs critères évocateurs & $14 \%$ \\
\hline Un critère évocateur & $28 \%$ \\
\hline Aucun critère retenu & $6 \%$ \\
\hline
\end{tabular}

Tableau 3. Répartition en fonction des critères du scorbut (Snoddy, 2018) / Distribution by diagnostic criteria for scurvy (Snoddy, 2018)

Trois individus présentent des lésions sur les grandes ailes des sphénoïdes, considérées comme un élément de forte probabilité de scorbut, pouvant ainsi supporter l'hypothèse initiale d'une population carencée (figures 3 et 4). D'après l'étude menée par Snoddy, et en suivant la classification en critères diagnostiques, évocateurs ou non, nous observons

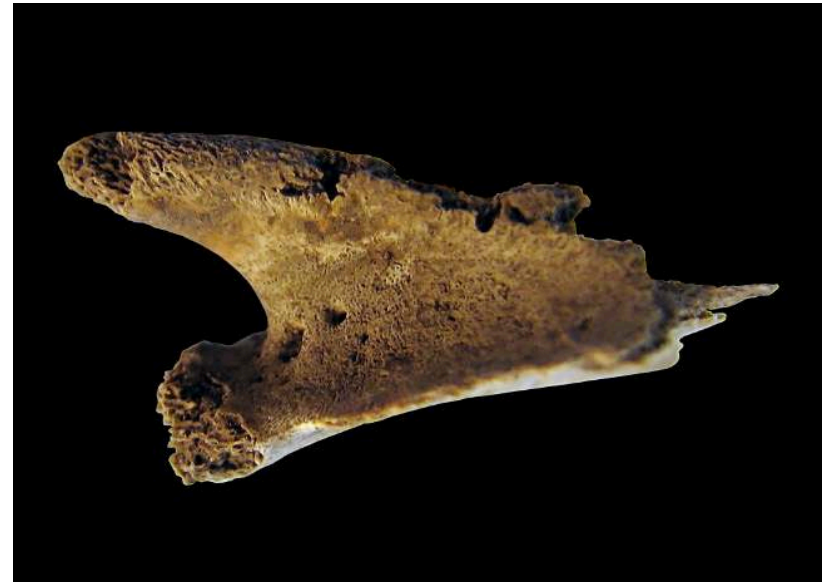

Figure 5. Scapula droite, individu 172, périnatal / Right scapula, skeleton 172, perinatal

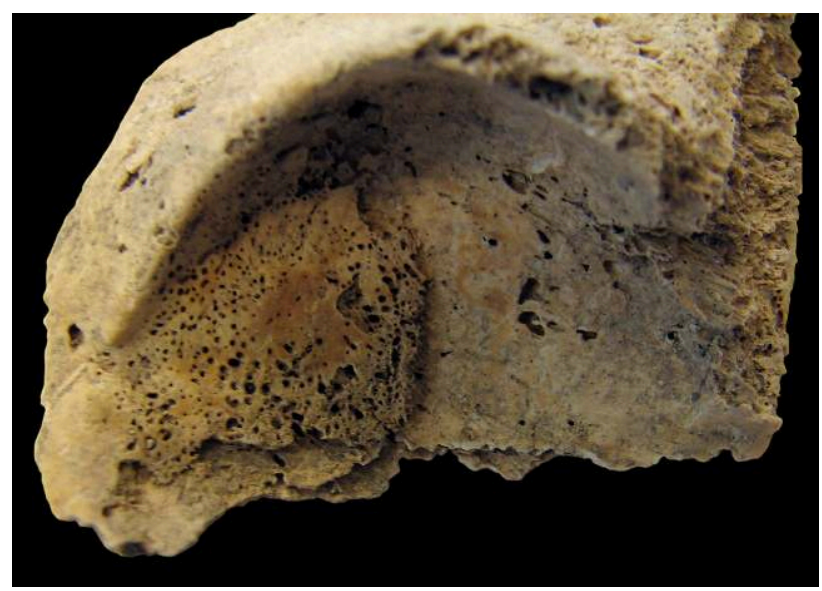

Figure 6. Orbite droit, individu 279, âgé entre 1 à 4 ans / Right orbital roof, skeleton 279, 1-4 year-old child

que deux tiers des immatures pathologiques présentent un ensemble de signes diagnostiques et/ou évocateurs du scorbut (tableaux 1 et 3 ; figures 5 et 6 ). Aucun cas évoquant du rachitisme n'a été identifié. Ce constat oriente encore un peu plus vers un contexte carentiel généralisé, qui mérite d'être discuté et recontextualisé.

\section{Discussion}

La nécropole de Jort est un site particulièrement remarquable, principalement en raison du nombre important d'immatures, tout à fait inhabituel parmi les ensembles funéraires étudiés à ce jour pour cette période. En effet, les sites funéraires connus sont plutôt caractérisés par de petits effectifs inférieurs à 50 individus et une très forte sous-représentation des sujets de moins de 5 ans (Chanson et al., 2010). De plus, la forte morbidité constatée dans cette collection ostéologique, avec $39 \%$ d'individus présentant des lésions pathologiques de même type, confère à cet ensemble une valeur informative majeure sur l'état sanitaire de la population infantile de Jort. 
Une limite interprétative mérite d'être soulignée, découlant du caractère non exhaustif de la fouille, comme cela est fréquemment le cas en contexte d'archéologie préventive. La partie centrale de la zone archéologique n'a pas encore fait l'objet de fouille à ce jour et nous impose donc la prudence quant aux conclusions. Cependant, nous pouvons déjà remarquer une distribution aléatoire des immatures sur les deux zones actuellement investiguées, sans concentration d'âge ou de pathologie, ce qui laisse supposer une homogénéité de répartition au sein de la nécropole, qui méritera d'être confirmée lors de fouilles futures.

Par ce recrutement tout à fait inhabituel, nous pouvons envisager plusieurs hypothèses, comme un choix spécifique de rassembler des immatures en un même lieu donné, ce qui serait un phénomène inédit pour la Normandie ; une surreprésentation relative liée à un autre mode de gestion funéraire des individus plus âgés comme la crémation ; ou bien une crise sanitaire durable responsable d'une surmortalité spécifique. Pour l'instant, aucun élément suffisamment tangible ne confirme ni n'infirme davantage l'une d'entre elles.

Les lésions pathologiques, à type de porosité corticale ou d'appositions périostées diffuses, sont fréquemment décrites chez les immatures, mais la proportion d'individus touchés à Jort est très supérieure aux taux habituellement publiés. La revue de la littérature paléopathologique montre que ce type d'atteintes est présent pour toutes les périodes, du Néolithique jusqu'à la période moderne, ainsi que sur la majeure partie des continents. Ces études ont généralement identifié moins de 20 cas, représentant au maximum $10 \%$ des immatures des séries ostéologiques, pour des sites qui ont été actifs durant plusieurs siècles (Ortner et al., 2001 ; Lovasz et al., 2013 ; Bourdou, 2014 ; Haagen, 2014 ; Krenz-Niebdala, 2016 ; Snoddy et al., 2017 ; Bourdou, 2018). Tous ces auteurs soulignent que ces lésions peuvent apparaître dans des situations étiologiques variées, telles que les infections, les traumatismes, ou les néoplasies, mais c'est le diagnostic de carence, et plus particulièrement le scorbut, qui est le plus fréquemment mis en avant dans ces contextes de cas multiples. Le diagnostic de scorbut est retenu à partir du moment où quelques individus présentent des lésions à localisations typiques (grande aile du sphénoïde, toit de l'orbite, scapula) et que le contexte historique concorde avec une période de trouble. Pour le site de Jort, la typologie des cas, la forte concentration d'individus touchés et une période d'occupation relativement courte, donnent une probabilité élevée à l'étiologie carentielle. Il semble effectivement difficile d'imputer l'ensemble de ces cas à une étiologie de type traumatisme, compte tenu de l'absence d'autre lésions traumatiques plus spécifiques comme les fractures. Les cancers de l'enfant ont une prévalence trop faible, avec une incidence annuelle actuelle estimée de 1,4 cas pour 10000 enfants de moins de 15 ans (Steliarova-Foucher et al., 2018). Les infections néonatales et infantiles, extrêmement fréquentes par ailleurs, mais d'évolution rapide, laissent rarement de traces osseuses. La comparaison avec les autres sites montre qu'avec $39 \%$ d'individus atteints, il s'agit d'une prévalence trop forte, au vu de nos connaissances actuelles, pour retenir un phénomène naturel, résultant d'un panachage de pathologies variées ayant une même expression.

L'étude du site de Kilkenny, en Irlande, représente un travail majeur pour la compréhension biologique et sociale de ce type de lésions. Il regroupe 970 individus décédés durant la grande famine irlandaise du milieu du $\mathrm{XIX}^{\mathrm{e}}$ siècle, et dont la moitié de la population présente ce type de lésions (Geber et Murphy, 2012). Cette collection ostéologique, avec $56 \%$ d'immatures, est un ensemble de référence d'une population décédée en contexte de carence alimentaire grave (Mays, 2014). La description méthodique et complète de l'ensemble des stigmates osseux, leurs fréquences, et leurs répartitions par classe d'âge, permettent d'identifier les conséquences osseuses d'un épisode de famine sur une population.

La comparaison de l'ensemble de Jort avec ce site montre un panel de lésions très similaires au site de Kilkenny. La proportion d'immatures touchés est certes inférieure au site irlandais (36\% pour $67 \%$ ) mais la mauvaise conservation des squelettes de Jort a pu engendrer une sous-estimation des cas. L'hypothèse d'un contexte de malnutrition infantile nous semble renforcée, grâce à la comparaison avec Kilkenny.

Peut-on alors retenir le scorbut comme étiologie commune à Jort ? Certes les critères paléopathologiques décrits pour retenir le diagnostic de scorbut sont encore aujourd'hui beaucoup discutés, imposant ainsi la plus grande prudence pour retenir ce diagnostic (Brickley et Ives, 2006 ; Brickley et Mays, 2019). Le scorbut est la maladie générée par la carence en vitamine $\mathrm{C}$, substance non métabolisable par l'être humain, et dépendant donc de son alimentation d'origine végétale. Compte tenu des réserves hépatiques capables de subvenir à nos besoins pendant deux à trois mois, une privation prolongée est donc nécessaire pour voir apparaître la symptomatologie (Akikusa et al., 2003). En conséquence, ce sont donc des contextes particuliers d'isolement ou de restriction qui sont à l'origine du scorbut.

Le contexte historique de Jort est relativement méconnu et encore sujet à discussion, mais certains éléments indiqueraient une période d'instabilité sociale. Certes, les commentaires de César rapportaient déjà des problèmes d'approvisionnement alimentaire à l'origine de conflits armés, comme notamment la révolte de Viridorix, qui intéresse le territoire proche de Jort (César, BG, III, 17). Les recherches actuelles menées à Port-en-Bessin-Huppain (Calvados) révèlent des preuves tangibles d'une occupation militaire romaine, dont l'envergure reste à déterminer, mais qui confirment l'instabilité politique à l'origine de révoltes persistantes en Gaule avant l'époque augustéenne (Goudineau, 1990 ; Lefort, 2015 ; Lefort et al., 2015 ; 2018). Par ailleurs, l'abandon de sites dotés d'une fonction commerciale importante au début de cette période, comme Urville-Nacqueville, peut être regardé comme un autre indicateur de cette instabilité (Lefort, 2015). L'apport de la dendrochronologie indique des périodes de sécheresse itératives, ayant une conséquence immédiate sur la production agricole (Durost et al., 2008). Dans ce contexte, une diminution possible de la production alimentaire et des difficultés d'approvisionnement sont envisageables, 
procurant un contexte favorisant l'apparition de famines et permettant de retenir, avec une bonne probabilité, le diagnostic de scorbut. Au-delà de ce diagnostic, c'est un tableau complet, associant un recrutement inhabituel, des lésions élémentaires rattachées à une pathologie carentielle, un profil lésionnel proche de celui d'un contexte de famine connu, et un contexte historique troublé, qui permet d'envisager un contexte de malnutrition infantile dans la région de Jort lors de la transition La Tène Finale/époque gallo-romaine.

\section{Conclusion}

Malgré les aléas de l'archéologie préventive, qui n'ont pas permis une exhaustivité du site, la nécropole de Jort, par sa taille, son recrutement inhabituel de très jeunes immatures et par sa période d'utilisation, revêt un intérêt majeur d'un point de vue anthropologique. La mise en évidence d'une forte proportion d'immatures présentant un profil paléopathologique évoquant un contexte carentiel, étayée par un faisceau d'arguments archéologiques et historiques de crise sociale, nous permet d'avancer l'hypothèse d'un épisode de famine. Un nouvel axe de recherche, impliquant l'augmentation du nombre de sites fouillés et un élargissement chronologique de l'étude, sera nécessaire pour toutefois confirmer et mesurer l'ampleur de ces premières conclusions.

\section{Liens d'intérêts}

Les auteurs déclarent ne pas avoir de liens d'intérêts.

\section{Références}

Adalian P (2001) Évaluation multiparamétrique de la croissance fœtale. Applications à la détermination de l'âge et du sexe. Thèse de doctorat, Université de la Méditerranée-Aix-Marseille II, $258 \mathrm{p}$

Akikusa J, Garrick D, Nash MC (2003) Scurvy: Forgotten but not gone. Journal of Paediatrics and Child Health 39:75-77 [https://doi.org/10.1046/j.1440-1754.2003.00093.x]

Bello S, Signoli M, Rabino Massa E, Dutour O (2002) Les processus de conservation différentielle du squelette des individus immatures. Implications sur les reconstitutions paléodémographiques. Bulletins et mémoires de la Société d'Anthropologie de Paris 14(3-4):245-262

Besnard-Vauterin CC (2011) Ifs, ZAC Object'Ifs sud : Habitat et lieux funéraires protohistoriques et vestiges antiques. Rapport final de fouille archéologique préventive. Inrap Grand Ouest, 3 vol., Caen

Bourdou C (2014) Evidence of childhood scurvy in a Middle Byzantine Greek population from Crete, Greece $\left(11^{\text {th }}-12^{\text {th }}\right.$ centuries AD). International Journal of Paleopathology 5:86-94 [https://doi.org/10.1016/j.ijpp.2013.12.002]

Bourdou C (2018) Life and death at the "The Land of Three Lakes": Revisiting the non-adults from Roman Aventicum, Switzerland ( $1^{\text {st }}-3^{\text {rd }}$ century CE). International Journal of Paleopathology 22:121-134 [https://doi.org/10.1016/j.ijpp.2018. 06.005]
Brickley M, Ives R (2006) Skeletal manifestations of infantile scurvy. American Journal of Physical Anthropology 129(2): 163-172 [https://doi.org/10.1002/ajpa.20265]

Brickley M, D’Ortenzio L, Kahlon B, Schattmann A, Ribot I, Raguin E, Bertrand B (2017) Ancient vitamin D deficiency: Long-Term Trends. Current Anthropology 58(3):420-427 [https://doi.org/10.1086/691683]

Brickley M, Mays S (2019) Metabolic Disease. In: Buikstra J (2019) Ortner's identification of pathological conditions in human skeletal remains, $3^{\text {rd }}$ edition, Academic Press, London, pp 531-566

Buikstra J (2019) Ortner's identification of pathological conditions in human skeletal remains, $3^{\text {rd }}$ edition, Academic Press, London, $839 \mathrm{p}$

Carpentier V (2015) Au cœur d'un village, nécropole d'enfants laténienne et occupations diachroniques, rapport final d'opération, Inrap Grand-Ouest, Normandie, 393 p

Chanson K, Delalande A, Jahier I et al (2010) Les pratiques funéraires au second âge du Fer en Basse-Normandie : premiers éléments de réflexion. In: Barral P, Dedet B, Delrieu F et al (dir) L'âge du Fer en Basse-Normandie. Gestes funéraires en Gaule au second âge du Fer. Actes du XXXIII ${ }^{\mathrm{e}}$ colloque international de l'AFEAF, Caen, 20 au 24 mai 2009, Besançon, Presses universitaires de Franche-Comté, pp 45-78

Durost S, Rossignol B, Lambert G (2008) Climat, Guerre des Gaules et dendrochronologie du chêne (Quercus sp.) du $\mathrm{I}^{\mathrm{er}}$ siècle av. J.-C. Revue d'archéométrie 32:31-50 [https://doi. org/10.4000/archeosciences.932]

Feugère M, Poux M (2001) Gaule pacifiée, Gaule libérée ? Enquête sur les militaria en Gaule civile. In: Deschler-Erb E et S (dir) Römisches Militär und Militaria im zivilen Umfeld - Roman Military Equipment in civil settlements: International Roman Military Equipment Conference (ROMEC XIII). Jahresbericht der Gesellschaft Pro Vindonissa, Vindonissa/ Brugg, pp 79-95

Garn S, Guzman M, Wagner B (1969) Subperiosteal gain and endosteal loss in protein-calorie malnutrition. American Journal of Physical Anthropology 30:153-156 [https://doi.org/10.1002/ ajpa.1330300120]

Geber J, Murphy E (2012) Scurvy in the Great Irish Famine: Evidence of vitamin C deficiency from a mid-19 ${ }^{\text {th }}$ century skeletal population. American Journal of Physical Anthropology 148:512-524 [https://doi.org/10.1002/ajpa.22066]

Goudineau C (1990) César et la Gaule, Paris, Errance, 365 p

Haagen K (2014) Subadult scurvy in Andean South America: Evidence of vitamin $\mathrm{C}$ deficiency in the late pre-Hispanic and Colonial Lambayeque Valley, Peru. International Journal of Paleopathology 5:34-45 [https://doi.org/10.1016/j.ijpp.2013. 09.002]

Lefort A, Rottier S (2014) Opérations de fouilles archéologiques sur l'estran d'Urville-Nacqueville. Rapport d'opération. La nécropole de la Tène finale. Rapport final d'opération de fouille programmée, SRA Normandie, Caen, volume 1, $67 \mathrm{p}$

Lefort A (2015) Contribution bas-normande à une archéologie des relations transmanches à la fin de l'âge du Fer. Échanges matériels et culturels. Thèse de doctorat, Université de Bourgogne-Franche-Comté, $353 \mathrm{p}$ 
Lefort A, Baron A, Blondel F et al (2015) Artisanat, commerce et nécropole. Un port de La Tène D1 à Urville-Nacqueville. In: Olmer F, Roure R (2015) Les Gaulois au fil de l'eau. Actes du $37^{\mathrm{e}}$ colloque international de l'AFEAF, Montpellier 2013, Ausonius, Bordeaux, pp 481-514

Lefort A, Marcigny C, Méniel P (2018) L'occupation militaire romaine préaugustéenne du mont Castel à Port-en-BessinHuppain et Commes (Calvados). In: Reddé M (2018) Les armées romaines en Gaule à l'époque républicaine. Nouveaux témoignages archéologiques, Glux-en-Brenne, Bibracte 28:207-248

Le Roux P (2004) La romanisation en question. Annales. Histoire, Sciences Sociales 59:287-311

Lovasz G, Schultz M, Gödde J et al (2013) Skeletal manifestations of infantile scurvy in a late medieval anthropological series from Hungary. Anthropological Science 121(3):173-185 [https://doi.org/10.1537/ase.130905]

Lewis M (2017) Paleopathology of Children. Identification of pathological conditions in the human skeletal remains of non-adults. Academic Press, London, $288 \mathrm{p}$

Mays S (2014) The palaeopathology of scurvy in Europe. International Journal of Paleopathology 5:55-62 [https://doi.org/ 10.1016/j.ijpp.2013.09.001]

Moorrees C, Fanning E, Hunt E (1963) Formation and resorption of tree deciduous teeth in children. American Journal of Physical Anthropology 21(2):205-213 [https://doi.org/10. 1002/ajpa.1330210212]

Ortner D, Bitler W, Cafarella J et al (2001) Evidence of probable scurvy in subadults from archeological sites in North America. American Journal of Physical Anthropology 114:343-353 [https://doi.org/10.1002/ajpa.1046]
Reddé M (2018) L'armée romaine en Gaule à l'époque républicaine. In: Reddé M (2018) Les armées romaines en Gaule à l'époque républicaine. Nouveaux témoignages archéologiques, Glux-en-Brenne, Bibracte 28:287-300

Snoddy A, Halcrow S, Buckley H et al (2017) Scurvy at the agricultural transition in the Atacama Desert (ca 3600-3200 BP): nutritional stress at the maternal-foetal interface? International Journal of Paleopathology 18:108-120 [https://doi.org/10.1016/ j.ijpp.2017.05.011]

Snoddy A, Buckley H, Elliot G et al (2018) Macroscopic features of scurvy in human skeletal remains: A literature synthesis and diagnostic guide. American Journal of Physical Anthropology 167:876-895 [https://doi.org/10.1002/ajpa.23699]

Steliarova-Foucher VA, Fidler M, Colombet M, et al (2018). Changing geographical patterns and trends in cancer incidence in children and adolescents in Europe, 1991-2010 (Automated Childhood Cancer Information System): a population-based study. The Lancet oncology 19(9):1159-1169 [https://doi.org/ 10.1016/S1470-2045(18)30423-6]

Toole MJ, Waldman RJ (1988) An analysis of mortality trends among refugee populations in Somalia, Sudan, and Thailand. Bulletin of the World Health Organization 66(2):237-247 [https://apps.who.int/iris/handle/10665/48418]

Vis H (1969) Protein deficiency disorders. Postgraduate Medical Journal 45:107-115 [http://dx.doi.org/10.1136/pgmj.45.520. 107]

Krenz-Niedbala M (2016) Did Children in Medieval and Postmedieval Poland Suffer from Scurvy? Examination of the Skeletal Evidence. International Journal of Osteoarchaeology 26:633-647 [https://doi.org/10.1002/oa.2454] 